\title{
Bio-inspired celestial compass yields new opportunities for urban localization
}

\author{
Julien Dupeyroux, Stéphane Viollet and Julien R. Serres
}

\begin{abstract}
Autonomous navigation requires multi-sensors data fusion provided either by global navigation satellite systems (GNSS) devices, inertial measurement units, radars and cameras to achieve accurate localization. Each technological solution features advantages but suffers also from drawbacks. Data fusion aims at maintaining a strong level of accuracy and robustness to make autonomous navigation systems reliable enough to be embedded on board any autonomous vehicles. However, there are still environmental contexts in which most sensors drift or even fail to provide correct estimates. In this study, we discuss the opportunity to use a celestial compass inspired by the desert ants' visual system which is able to extract heading information from the polarization pattern of the skylight in the ultraviolet (UV) spectrum. This new sensing mode has been mounted on-top a car and tested outdoor on a 18.6km-long journey in town and compared with GNSS estimates. The UV celestial compass yielded promising performances regarding its low complexity and the root mean square error of the orientation error was only $0.55^{\circ}$. Our results suggest the suitability of such parsimonious insect-inspired solutions for robotic purposes in urban field like the last mile delivery.
\end{abstract}

\section{INTRODUCTION}

Autonomous navigation is one of the most exciting technical challenges of the 21 st century. More than 5000 container ships cross the seas every year, and more than 5000 aircraft are flying at any given time. But it is nothing compared to the billion of vehicles circulating around the world. Besides, the fast development of autonomous drones and ground vehicles involves new technological challenges in navigation. Localizing these systems benefits from a vast number of methods, mostly relying on civilian global navigation satellite systems (GNSS), inertial measurement units (IMU), radars and cameras [1], [2]. These systems must achieve centimetre precision and robust localization estimates to ensure their suitability for large scale applications like long range urban navigation.

Since its introduction to civilian applications in the 1980s, the global positioning system (GPS) has been largely deployed on-board vehicles, robots and smart devices, with a global average position accuracy below $7 m$ (horizontal axis) and $15 m$ (vertical axis) according to recent investigations [3]. Although GPS-based methods play a key role in outdoor self-localization, they still remain prone to uncertainty as the satellite coverage might be poor, the signal can be disturbed

*This research was supported by the French Direction Générale de l'Armement (DGA), CNRS, Aix Marseille University, the Provence-AlpesCôte d'Azur region, and the French National Research Agency for Research (ANR) with the Equipex/Robotex project.

All authors are with the Biorobotics Team, Aix Marseille Univ, CNRS, ISM, Marseille, France julien. serresduniv-amu. fr by the atmosphere - thus resulting in high latency and/or deteriorated signal-to-noise ratio - or even obstructed by the buildings in urban canyons.

Other navigation techniques comprise the use of visual odometry (VO). The fast development of camera-based strategies, like the simultaneous and localization mapping (SLAM) ones, has led to outstanding results on-board cars, drones, and other ground vehicles for outdoor and indoor applications [4], [5], [6]. However, the use of traditional cameras has raised specific limitations as the processing chain requires high computational resources although the frame rate is low; besides, outdoor applications are severely impacted by the ambient light variations (ex: sunlight glare at the exit of tunnels) [7], [8]. Event-based cameras (DVS, ATIS, DAVIS) could prevent the lighting dependency and therefore achieve more robust VO [9], [10]. Another advantage of using such sensors would be the higher acquisition rate (up to $2 \mathrm{kHz}$ ) and low latency (in the range of the microsecond), due to the asynchronous detection of the moving contrasts. High-resolution, real-time VO can also be performed by laser detection (LIDAR); again, such techniques imply heavy computing limiting their use on board robots [11].

Indirect estimates of navigation paths can be obtained through proprioceptive sensors such as accelerometers and gyroscopes within IMUs. Combining these sensors with GNSS measurements can increase the overall accuracy of the localization [12]. Besides, IMUs are rather cheap, small and accessible. Although the long-term drifts of both accelerometers and gyroscope are easy to filter (ex: using Kalman filters), their sensitivity to electromagnetic interferences, especially for capacitive accelerometers, is a strong restriction for urban applications.

In short, there exists a wide range of technological solutions to make a robot autonomously navigating. Yet, the performances highly fluctuate with the environment itself, like in urban canyons or in areas with poor satellite coverage or high electromagnetic interference. It is therefore essential that we develop new sensing modes offering redundancy in self-localization to ensure more robust and accurate position estimates.

Drawing inspiration from navigating insects could provide parsimonious solutions as these tiny animals perform every-day long-distance navigation upon which their survival depends, with few resources and in complex even hostile environments. Desert ants Cataglyphis fortis are among the best navigators: every day, they rely on visual cues to forage up to $1 \mathrm{~km}$ before homing! Studies on navigating insects, including desert ants, bees, crickets, locusts and monarch 

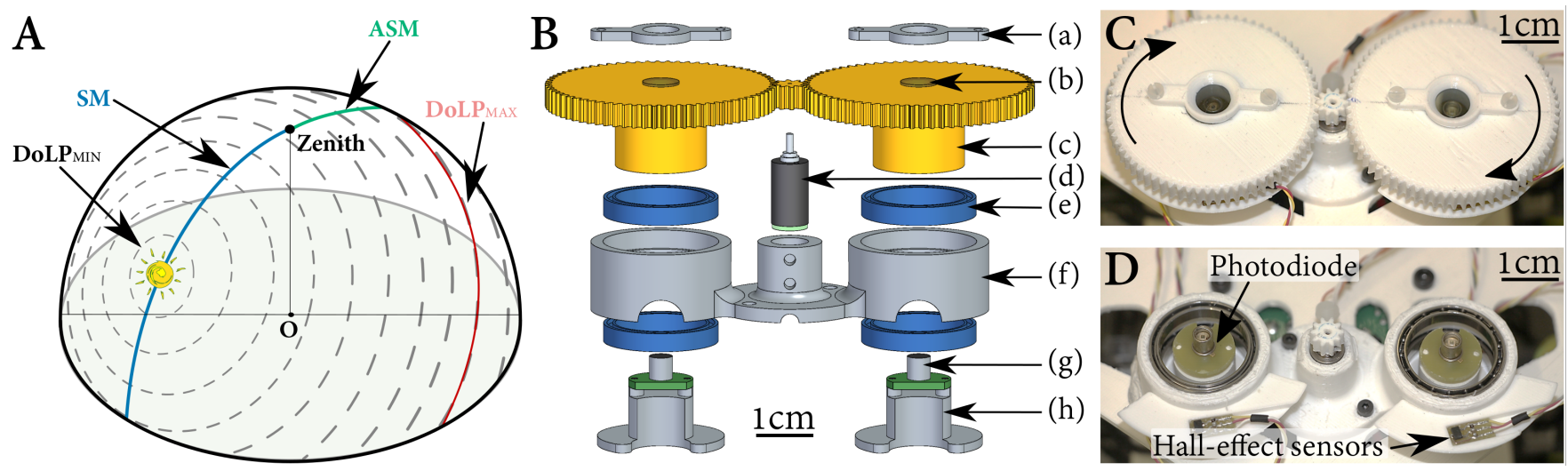

Fig. 1. A Polarization pattern of the skylight across the sky dome. The bars depict the state of polarization of the light as seen by an observer $\mathrm{O}$ (orientation: AoP; thickness: DoLP). SM: solar meridian; ASM: anti-solar meridian; DoLP: degree of linear polarization. Around the zenith, the AoP is orthogonal to the solar meridian. B Exploded computer-aided design (CAD) view of the celestial compass. (a) Fixation. Support used to hold the UV sheet polarizer. (b) UV linear sheet polarizer. (c) Rotating gears. (d) Stepper motor. (e) Ball bearings. (f ) Main frame of the celestial compass. (g) UV-sensitive photodiodes. (h) Support for UV-light sensors PCB. C,D Photographs of the sky compass. Adapted from [25].

butterflies, have shown that they detect the polarization state of skylight, mostly in the ultraviolet (UV) range [13]. According to the Rayleigh diffusion of the skylight, the sun produces a celestial pattern of linear polarization across the sky dome (Fig. 1A). The polarization state of light is defined by the angle of polarization (AoP), i.e. the orientation in which the light wave oscillates, and the degree of linear polarization (DoLP) corresponding to the ratio between the intensity of the linearly polarized light to the total ambient light intensity. Both the AoP and the DoLP depend on multiple parameters like the spectral wavelength and the composition of the atmosphere, but also on the position of the sun, the position of the observer, and the part of the sky dome that is observed. The photoreceptors located in the dorsal rim area of the insects' compound eyes are sensitive to specific AoPs [14]. The neural pathway within these insects' brains have been partially identified, and can now be considered for robotic simulations and implementations for navigation applications.

In the late 1990s, Lambrinos et al. introduced the first implementation of an insect-inspired celestial compass, composed of three static polarization units sensitive to linear polarized skylight in the blue range [15]. Tests conducted in the desert resulted in a heading angle error of $0.66^{\circ}$. However, such performance should be qualified in that the experimental set-up offered optimal conditions as the compass has been tested early in the morning and late in the afternoon, when the DoLP is maximum at the zenith (Fig. 1A). More recently, Chu et al. designed and built several miniaturized version of Lambrinos' sensor [16], [17]. Results revealed high precision in controlled light, with average orientation error as low as $0.1^{\circ}$ when applying polynomial fitting. Other insectinspired sensors have been developed [18], [21], [19], [20], although none of them were tested on fully autonomous navigation tasks. Incidentally, the vast majority of these robotic implementations of the insects' visual system did not opt for spectral sensitivity in the UV range, which is not fully consistent with biological findings.
In 2018, Fan et al. investigated on the use of their celestial compass for car navigation applications [22], [23]. Their polarized sensor combined four polarization units made of CCD cameras $(1034 \times 778$ pixels $)$ with wide-angle lens and spectral sensitivity in the visible range $\left(\lambda_{\max }=650 \mathrm{~nm}\right)$. Once embedded on board the car, the sensor was tested on a $2.14 \mathrm{~km}$-long trajectory, resulting in an orientation root means square error (RMSE) equal to $0.81^{\circ}$. In 2017, Wang et al. investigated the effect of foliage on the AoP measurements in car navigation [24] using the same polarized sensor as Fan and colleagues [22], [23]. Their method was tested on a $2.52 \mathrm{~km}$-long journey, resulting in an orientation RMSE equal to $0.92^{\circ}$ despite foliage. Recently, Dupeyroux et al. demonstrated the superiority of the AoP detection in the UV range under foliage, whose measurement depends mainly on meteorological conditions (clear sky: $0.39^{\circ}$; covered sky: $0.59^{\circ}$, using a median filter) [26].

The present study aims at introducing an UV insectinspired celestial compass for the determination of the heading of a mobile ground vehicle in town over a long-range trajectory. Section 2 presents the celestial compass and how to use it for heading estimation purposes. Three distinct methods are considered, namely a GPS-based approach, and two methods relying on the celestial compass: the AntBot method [25], [26], and the Hilbert method, introduced in this study. Section 3 describes the results obtained over a $18.6 \mathrm{~km}$ long journey in town. Results show that our UV-sensitive celestial compass yields a RMSE error of only $0.55^{\circ}$ using the AntBot method, ca. half the value of previous works with only 2 pixels.

\section{MATERIAL AND METHODS}

\section{A. The celestial-based heading estimation}

We developed an ant-inspired sky compass [25], [26] (Fig. 1B-D) that analyses the polarization pattern of the skylight to extract the AoP around the zenith, corresponding to the heading of the vehicle with respect to the sun. The sensor comprises only two UV-sensitive photodiodes (SG01D- 
18 from SgLux GmbH, Berlin, Germany, $\lambda_{\max }=280 \mathrm{~nm}$ ) topped with linear sheet polarizers (HNP'B replacement from Knight Optical Ltd., Harrietsham, UK). The spectral sensitivity of the photodiode-polarizer combination provides a peak response at $\lambda_{\max }=343 \mathrm{~nm}$, which is close to maximum sensitivity at $350 \mathrm{~nm}$ in desert ants Cataglyphis [27]. These filters are made rotating to scan the polarization pattern around the zenith, each UV-pixel generating $N$ measurements per filter turn. The whole system is fully $3 \mathrm{D}$-printed and open-source $^{1}$. The two polarization units, called $U V_{0}$ and $U V_{1}$, are arranged orthogonally to each other, i.e. the phase difference between each polarizer is equal to $90^{\circ}$.

1) The AntBot method [25], [26]: the estimation of the heading angle requires to carry out a complete rotation of the polarizers, which lasts for 4 seconds considering an angular resolution of $\Delta \psi=360^{\circ} / 224=1.61^{\circ}(N=224)$. Due to the symmetry of the polarization pattern across the sky dome, the signals measured by the sky compass are $180^{\circ}$-periodic sine waves with a phase opposition. The two raw signals, coming from each UV-sensitive photodiode, are low pass filtered and normalized before AoP computation. The two filtered signals $U V_{0}$ and $U V_{1}$ can be mathematically expressed as follows:

$$
\left\{\begin{array}{l}
U V_{0, i}(\psi)=\frac{1}{2+\varepsilon}\left(1+\cos \left(2\left(\psi+\psi_{i}\right)\right)+\varepsilon\right) \\
U V_{1, i}(\psi)=\frac{1}{2+\varepsilon}\left(1+\cos \left(2\left(\psi+\psi_{i}+90^{\circ}\right)\right)+\varepsilon\right)
\end{array}\right.
$$

where $\psi$ is the AoP, $\psi_{i}$ is the orientation of the polarizers for $i \in\{1, \ldots, N\}$, and $\varepsilon=10^{-6}$ is set to prevent from logratio computation failure (division by zero). Consequently, $U V_{0}$ and $U V_{1}$ are both constrained between $\varepsilon /(2+\varepsilon)$ and 1 .

The insect-inspired method of AoP computation estimates the AoP $\psi$ (Eq. 2) from the $\log$-ratio $P$ (Eq. 4) of the two POL-units' pre-processed signals [26]:

$$
\psi=\frac{1}{2}\left(\psi_{M I N}+\psi_{M A X}+\operatorname{sign}\left(\psi_{1}-\psi_{2}\right) \cdot 90^{\circ}\right)
$$

where $\psi_{M I N}$ and $\psi_{M A X}$ are given by:

$$
\left\{\begin{array}{lll}
\psi_{M I N}=\Delta \psi \cdot \arg \min _{i \in\{1, \ldots, N\}} P_{i} & \bmod 180^{\circ} \\
\psi_{M A X}=\Delta \psi \cdot \arg \max _{i \in\{1, \ldots, N\}} P_{i} & \bmod 180^{\circ}
\end{array}\right.
$$

where for all $i \in\{1, \ldots, N\}$ :

$$
P_{i}=\log _{10}\left(\frac{1+\cos \left(2\left(\psi+\psi_{i}\right)\right)+\varepsilon}{1-\cos \left(2\left(\psi+\psi_{i}\right)\right)+\varepsilon}\right)
$$

This method has been previously investigated and resulted in outstanding performances with a strong resilience toward both the meteorological and environmental conditions [26], [28]. The results also suggested that applying a median filter would have an interesting impact on the final heading estimation. This filtering process has been added in this study to improve the vehicle's heading estimation.

${ }^{1}$ https://github.com/JuSquare/AntBot/tree/master/CelestialCompass
2) Use of the Hilbert transform: based on the POL-units' pre-processed signals $U V_{0}$ and $U V_{1}$ (Eq. 1), the Hilbert transform was computed to determine the AoP. Basically, we compute the Hilbert transforms $\hat{U V} V_{0} \hat{U V} V_{1}$ of the two POL-units using the MATLAB function hilbert. We also computed the two corresponding $\pi$-periodic reference sine waves defined as follows:

$$
\left\{\begin{array}{l}
\hat{s}_{0, r e f}=\operatorname{hilbert}(\sin (\pi t)) \\
\hat{s}_{1, \text { ref }}=\operatorname{hilbert}(\sin (\pi t+\pi))
\end{array}\right.
$$

$t$ is the time vector, ranging from $0.018(4 / 224)$ to 4 seconds with a time step of 0.018 second. We then determine the AoP $\psi$ as follows:

$$
\psi=\frac{1}{2}\left(\psi_{0}+\psi_{1}\right)
$$

where:

$$
\left\{\begin{array}{l}
\psi_{0}=\text { mean }\left(\operatorname{angle}\left(\hat{U V} V_{0} * \operatorname{conj}\left(\hat{s}_{0, r e f}\right)\right)\right) \\
\psi_{1}=\text { mean }\left(\operatorname{angle}\left(\hat{U V} V_{1} * \operatorname{conj}\left(\hat{s}_{1, r e f}\right)\right)\right)
\end{array}\right.
$$

Here, $*$ refers to the Hadamard product (i.e. element-wise multiplication).

\section{B. GNSS-based methods}

The GNSS module used in this study is the $\mathrm{XL} \mathrm{M}^{2} \mathrm{P}^{2}$ from Drotek, based on u-blox NEO-M8P-2 chip (rover mode, Fig. 2B). The I/O data supported protocols are RTCM3, UBX, and NMEA. We used two distinct GN-based messages to determine the vehicle's heading:

- From GNGGA messages, we extracted the latitude and longitude of the vehicle and converted them into Cartesian system as an approximation for small areas. We then computed the heading of the vehicle based on the $(x, y)$ coordinates.

- From GNRMC messages, we simply extracted the orientation of the vehicle.

The GN prefix means that the received message is a mix of GPS and GLONASS satellite navigation systems.

\section{The experimental set-up}

The celestial compass and the GNSS unit were placed on a car top (Fig. 2A,B). Both sensors were connected to a Raspberry Pi 2B microcomputer, also connected to a laptop via RJ45 cable. Data were collected over several boulevards in Marseille, France. The GNSS unit provided one sequence of messages per second ( $\left.f_{G N S S}=1.0 \mathrm{~Hz}\right)$, including $G N G G A$ and GNRMC messages; and the celestial compass provided one full acquisition every 4 seconds $\left(f_{C O M P}=0.25 \mathrm{~Hz}\right)$. The boulevards comprise large old trees partially covering the road, and tall buildings. The $18.6 \mathrm{~km}$-long journey is detailed in Fig. 2C and consisted in three distinct roads: the Luminy road, a sinuous road equally distributed between the National Park of Calanques and the city with a variation of altitude equal to 110 meters, and two straight boulevards named Michelet (variation of altitude: 25 meters) and Prado.

\footnotetext{
${ }^{2}$ https://store.drotek.com/DP0503
} 
The experiments were conducted during the afternoon under sunny weather conditions.
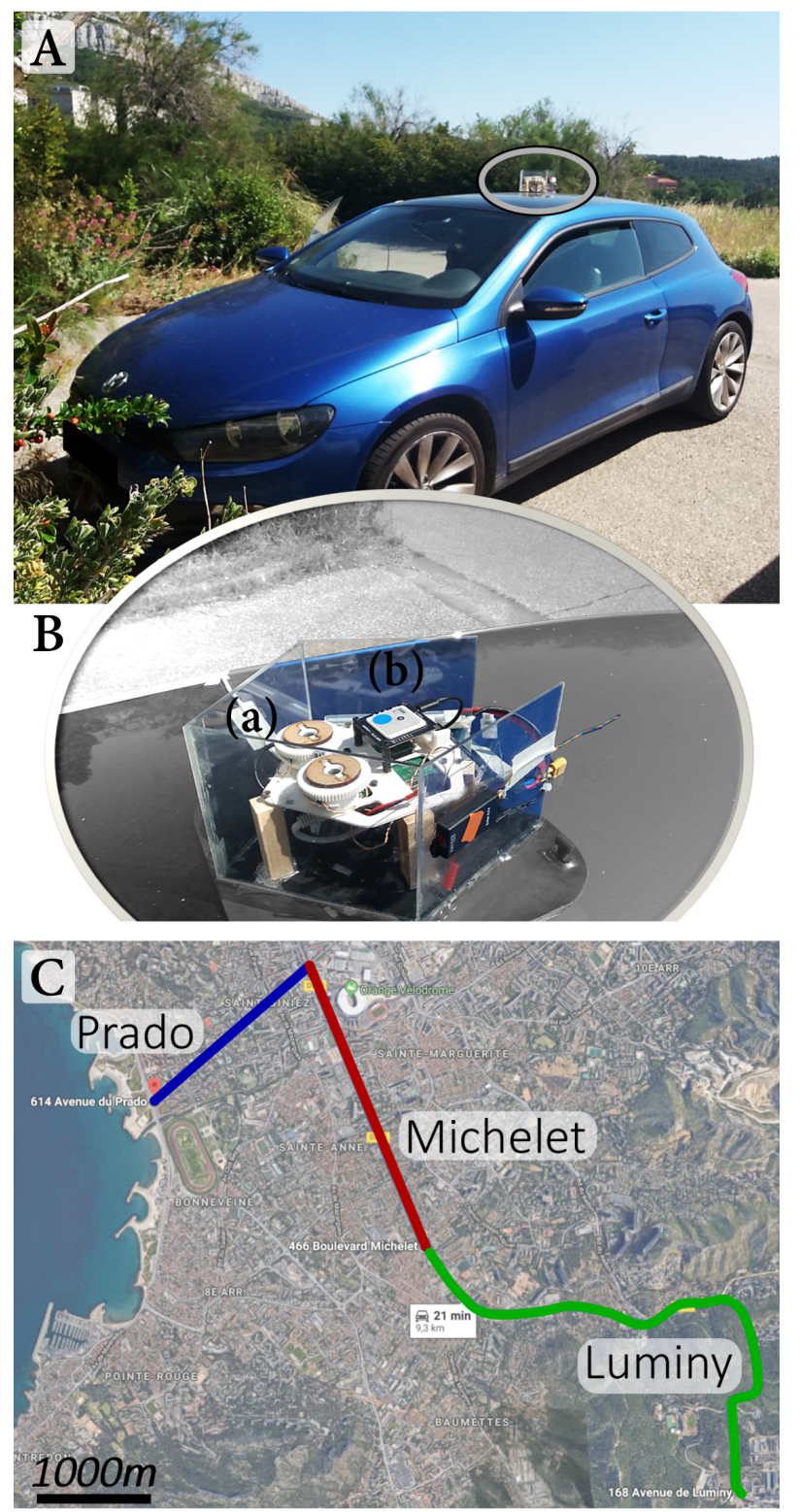

Fig. 2. The experimental set-up. A The sensors were fixed magnetically on the car top. B Magnified view of (a) the celestial compass and (b) the GNSS module. C Aerial view of the city of Marseille, France, including the testing trajectory which was composed of Luminy road (green), and both Michelet (red) and Prado (blue) boulevards.

\section{RESULTS}

Between June 2018 and June 2019, two data sets were collected in Marseille, France. In the first one, the setup parameters resulted in $f_{C O M P}=1 / 13=0.077 \mathrm{~Hz}$ for a corresponding angular resolution $\Delta \psi=360^{\circ} / 374=0.96^{\circ}$. In the data set acquired in 2019 , the polarization measurement has been upgraded to $f_{C O M P}=1 / 4=0.25 \mathrm{~Hz}$, resulting in a lower resolution $\Delta \psi=360^{\circ} / 224=1.61^{\circ}$.

First, the ability of our sky compass to accurately determine the vehicle's heading on a straight road has been investigated over the 2019 data set. The results for GNRMC messages and the two sky compass-based methods are displayed in Fig. 3 and in table I. For sake of clarity, results obtained with GNGGA messages were not plotted (standard deviations (SD) ranged between $35.98^{\circ}$ and $55.79^{\circ}$ ). GNRMC messages led to poor results during the outbound trajectory, with median error of $9.08^{\circ}$ and SD greater than $20^{\circ}$, while the celestial-based methods yielded absolute median errors less than or equal to $3.8^{\circ}$. During the inbound trajectory, the estimation of the heading angle resulted in median errors of only $0.78^{\circ}$ and $-0.35^{\circ}$ for the AntBot and the Hilbert methods, respectively. We also computed the RMSE, in degrees, to determine how well each method predicts the heading angle of the moving vehicle. The AntBot method provided RMSE as low as $0.55^{\circ}$ (outbound) and $0.61^{\circ}$ (inbound), which is lower than the angular resolution of the celestial compass $\left(\Delta \psi=1.61^{\circ}\right)$. However, the poor performances of the GNSS-based methods must be treated with caution as signal failures occurred $85 \%$ of the time. On the inbound trajectory, all methods, excepted the one based on GNGGA messages, were found statistically non-different at the default 5\% significance level according to Wilcoxon ranksum test.

TABLE I

HEADING ESTIMATION PERFORMANCES OVER MiCHELET BOULEVARD (2019 DATASET). SD: STANDARD DEVIATION.

\begin{tabular}{lllccc}
\hline Route & \multicolumn{2}{c}{ Method } & Median error & SD & RMSE \\
\hline \multirow{2}{*}{ Out } & \multirow{2}{*}{ GPS } & RMC & $9.08^{\circ}$ & $20.22^{\circ}$ & $2.47^{\circ}$ \\
& & GGA & $-24.12^{\circ}$ & $55.79^{\circ}$ & $4.91^{\circ}$ \\
& \multirow{4}{*}{ Compass } & AntBot & $-2.51^{\circ}$ & $6.81^{\circ}$ & $0.55^{\circ}$ \\
& & Hilbert & $3.80^{\circ}$ & $14.62^{\circ}$ & $1.19^{\circ}$ \\
\hline \multirow{2}{*}{ In } & GPS & RMC & $0.44^{\circ}$ & $2.86^{\circ}$ & $0.35^{\circ}$ \\
& \multirow{4}{*}{ Compass } & GGA & $-15.41^{\circ}$ & $35.98^{\circ}$ & $3.73^{\circ}$ \\
& & AntBot & $0.78^{\circ}$ & $6.50^{\circ}$ & $0.61^{\circ}$ \\
& & Hilbert & $-0.35^{\circ}$ & $5.98^{\circ}$ & $0.57^{\circ}$ \\
\hline
\end{tabular}

According to the first dataset collected in June 2018, we compared the heading estimation on the two boulevards Michelet and Prado, forming an absolute angle of approximately $80^{\circ}$. Results for GNGGA and the AntBot methods are presented in Fig. 4. All results are given modulo $180^{\circ}$. On the Michelet Boulevard, the measured heading was equal to $9.91^{\circ} \pm 0.67^{\circ}$ (mean $\pm \mathrm{SD}$ ) with the GNGGA-based strategy, and to $8.33^{\circ} \pm 1.74^{\circ}$ with the AntBot method. Then, when on the Prado boulevard, the GNSS-based heading was equal to $113.04^{\circ} \pm 0.98^{\circ}$, and the sky compass provided a mean angle of $111.96^{\circ} \pm 2.64^{\circ}$. All methods gave statistically non-different results according to Wilcoxon ranksum test $(P>0.05)$.

Both GNSS- and celestial-based methods provided an angular shift equal to $103^{\circ}$ (modulo $180^{\circ}$ ) between the two boulevards, which is equivalent to $-77^{\circ}$ if we respect the clockwise rule. This finding is consistent with the initial expectations on the angular shift modulo $180^{\circ}$. Besides, comparing the distributions of estimated headings, no significant difference was found between the two methods for each tested boulevard (Fig. 4). 


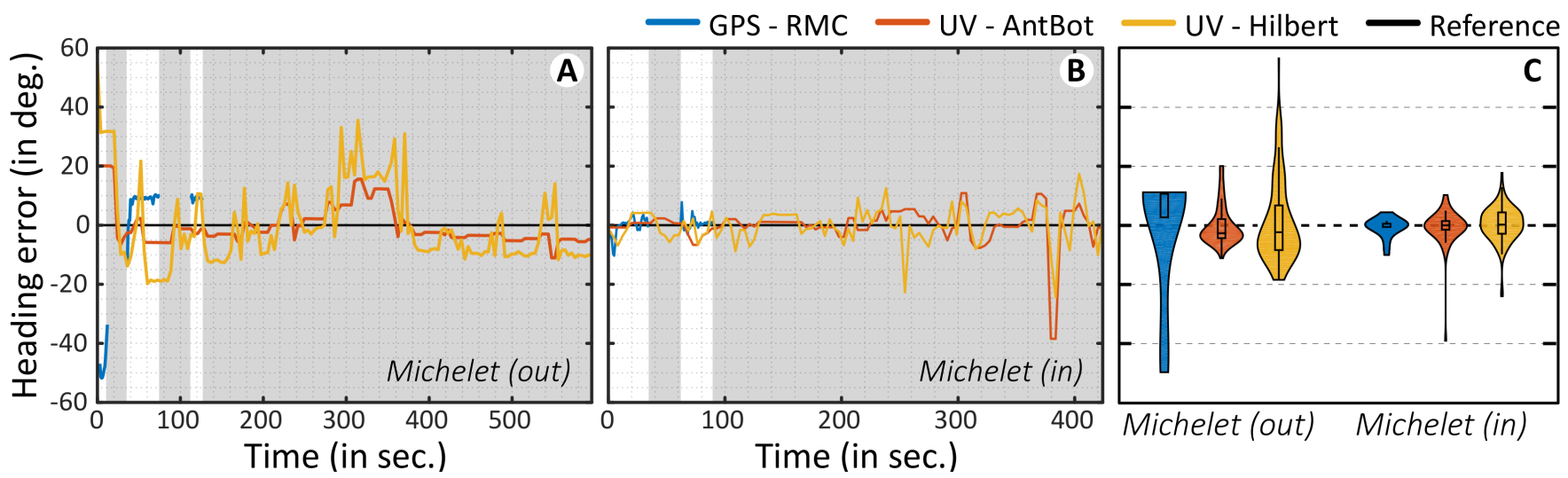

Fig. 3. Results obtained on Michelet boulevard according to the second dataset (06-2019; $f_{C O M P}=0.25 H z$ ). A-B Heading error (in degrees) in the back and forth direction. Gray areas depict the moments when the GPS was unavailable. C Corresponding box and violin plots of the heading error, showing the probability density corresponding to each error value. The heading reference, used for error estimation, is obtained from the geographic directions provided by maps.
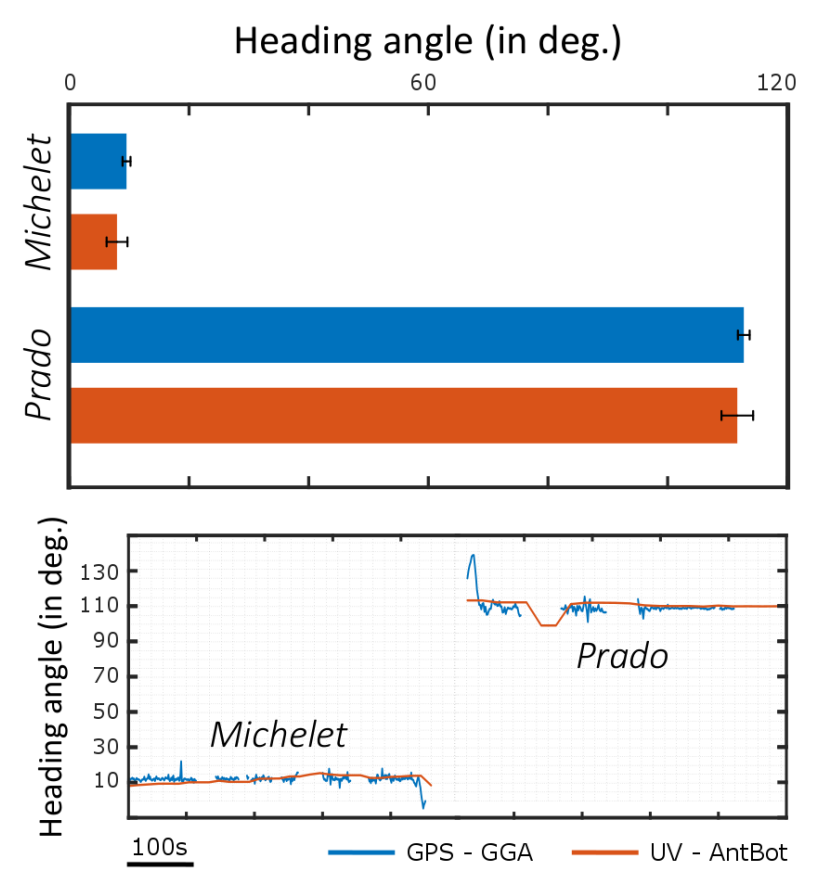

Fig. 4. Estimation of the heading angle over two straight boulevards shifted by $-80^{\circ}$ (i.e. $100^{\circ} ; 2018$ dataset).

Lastly, we investigated on the performances of the heading estimation on sinuous roads (e.g. the Luminy road; 2018 dataset; Fig. 5B). Results are displayed in Fig. 5A. At the begining of the trajectory, the vehicle's heading often changed rapidly, producing important variations in the estimations between $t=0 \mathrm{~s}$ and $t=250 \mathrm{~s}$. In this time interval, we notice that the curves follow the same variations, apart from the AntBot method which gave opposite results. However, beyond $t=250 \mathrm{~s}$, i.e. when the road gets smoother, all the methods led to homogeneous heading estimations.

\section{CONCLUSION AND FUTURE WORK}

The use of a 2-pixel, UV-sensitive and insect-inspired celestial compass for the determination of a vehicle's ori-
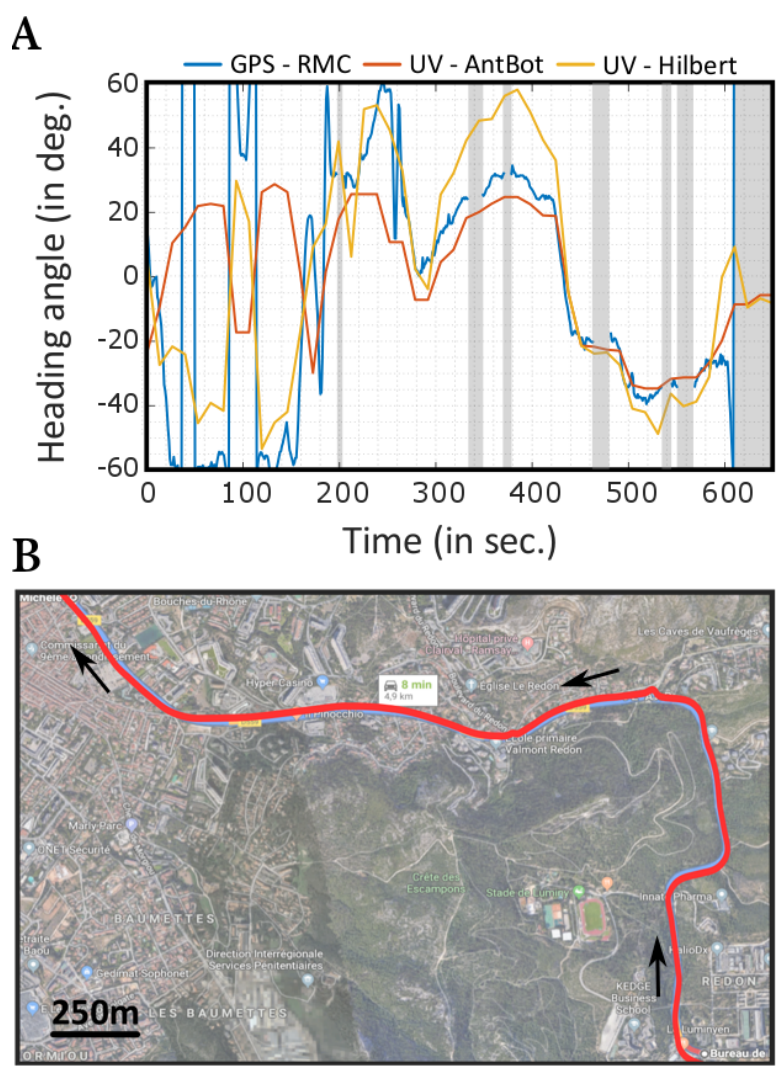

Fig. 5. A Heading angle (in degrees) estimated over the Luminy road, according to the first dataset $\left(06-2018 ; f_{C O M P}=0.077 \mathrm{~Hz}\right)$. Gray areas depict the moments when the GPS was unavailable. B Sky view of the trajectory.

entation during urban navigation has yielded low RMSE of $0.55^{\circ}$ with the AntBot method. Through this study, we confirmed the great potential of applying an insectinspired polarization-sensitive optical compass to outdoor autonomous localization, especially in the UV-range. Once merged with other navigation systems, this insect-inspired sky compass could help maintaining the localization of the vehicle in case of failure from other sensors. 
In particular, we observed that GNSS failure occured at a ratio up to $85 \%$ of the acquisition time. These results were obtained over two main boulevards of the city, highly prone to heavy traffic, and where the risk of GNSS signal occlusion is important due to the presence of buildings and trees.

Further investigations will focus on two major axes. First, there is a need for a real-time version of the sky compass, which will obviously involve a static measurement of the polarization pattern (i.e. without any actuation of the polarizer sheets). Static celestial compasses already exist [15], [17], but they only include 3 POL-units and we have shown in [28] that these configuration generally lead to poor performances in adverse weather conditions. Recently, Polaris Sensor Technologies Inc. has developed its own celestial compass, called SkyPASS [29], which is based on pattern polarization matching. However, the SkyPASS accuracy strongly depends on both weather conditions and foliage. Consequently, AntBot's proof-of-concept has been patented for car localization in order to be accurate despite cloudy conditions and foliage, but also real-time acquisition by using it in its static version [30].

On the other hand, the spectral sensitivity represents a key element in making this technology suitable for automotive applications. UV-friendly sensors and polarizers are quite expensive (about 100 euros per $\mathrm{cm}^{2}$ ). Although most navigating insects detect the polarization pattern in the UV range, other insects like bees and crickets acquire those information in the blue or in the green range. Since linear polarizers and photodiodes in the visible range are cheaper, a trade-off between the spectral sensitivity, the navigation performance and the production price should be reached by combining different spectral channels.

\section{ACKNOWLEDGEMENT}

The authors would like to thank Marc Boyron and Julien Diperi for their technical support.

\section{REFERENCES}

[1] S. A. Mohamed, M. H. Haghbayan, T. Westerlund, J. Heikkonen, H. Tenhunen, and J. Plosila, A Survey on Odometry for Autonomous Navigation Systems, IEEE Access, vol. 7, pp. 97466-97486, 2019.

[2] I. Skog, and P. Handel, In-car positioning and navigation technologies A survey, IEEE Transactions on Intelligent Transportation Systems, vol. 10, no. 1, pp. 4-21, 2009.

[3] B. A. Renfro, M. Stein, N. Boeker, and A. Terry, An analysis of global positioning system (GPS) standard positioning service (SPS) performance for 2017, 2019.

[4] M. J. Milford, and G. F. Wyeth, SeqSLAM: Visual route-based navigation for sunny summer days and stormy winter nights, IEEE International Conference on Robotics and Automation, pp. 1643-1649, 2012.

[5] S. Weiss, D. Scaramuzza, and R. Siegwart, Monocular-SLAM-based navigation for autonomous micro helicopters in GPS-denied environments, Journal of Field Robotics, vol. 28, no. 6, pp. 854-874, 2011.

[6] Y. Ham, K. K. Han, J. J. Lin, and M. Golparvar-Fard, Visual monitoring of civil infrastructure systems via camera-equipped Unmanned Aerial Vehicles (UAVs): a review of related works, Visualization in Engineering, vol. 4, no. 1, p. 1, 2016.

[7] S. Lowry, and M. J. Milford, Supervised and unsupervised linear learning techniques for visual place recognition in changing environments. IEEE Transactions on Robotics, vol. 32, no. 3, pp. 600-613, 2016.
[8] A. R. Vidal, H. Rebecq, T. Horstschaefer, and D. Scaramuzza, Ultimate SLAM? Combining events, images, and IMU for robust visual SLAM in HDR and high-speed scenarios, IEEE Robotics and Automation Letters, vol. 3, no. 2, pp. 994-1001, 2018.

[9] E. Mueggler, H. Rebecq, G. Gallego, T. Delbruck, and D. Scaramuzza, The event-camera dataset and simulator: Event-based data for pose estimation, visual odometry, and SLAM. The International Journal of Robotics Research, vol. 36, no. 2, pp. 142-149, 2017.

[10] G. Gallego, T. Delbruck, G. Orchard, C. Bartolozzi, B. Taba, A. Censi, S. Leutenegger, A. Davison, J. Conradt, K. Daniilidis, and D. Scaramuzza, Event-based vision: A survey. arXiv preprint arXiv:1904.08405, 2019.

[11] M. Fallon, Accurate and robust localization for walking robots fusing kinematics, inertial, vision and LIDAR, Interface focus, vol. 8, no. 4, 20180015, 2018.

[12] Y. Zhao, Applying time-differenced carrier phase in nondifferential GPS/IMU tightly coupled navigation systems to improve the positioning performance. IEEE Transactions on Vehicular Technology, vol. 66, no. 2, pp. 992-1003, 2016.

[13] R. Wehner, and S. Wehner, Insect navigation : use of maps or Ariadne's thread?, Ethology Ecology \& Evolution, vol. 2, no. 1, pp. 2748, 1990.

[14] T. Labhart, and E. P. Meyer, Detectors for polarized skylight in insects: a survey of ommatidial specializations in the dorsal rim area of the compound eye, Microscopy Research and Technique, vol. 47, no. 6, pp. 368379, 1999.

[15] D. Lambrinos, H. Kobayashi, R. Pfeifer, M. Maris, T. Labhart, and R. Wehner, An autonomous agent navigating with a polarized light compass. Adaptive behavior, vol. 6, no. 1, pp. 131-161, 1997.

[16] J. Chu, K. Zhao, Q. Zhang, and T. Wang, Construction and performance test of a novel polarization sensor for navigation. Sensors and Actuators A: Physical, vol. 148, no. 1, pp. 75-82, 2008.

[17] J. Chu, Z. Wang, L. Guan, Z. Liu, Y. Wang, and R. Zhang, Integrated polarization dependent photodetector and its application for polarization navigation. IEEE Photonics Technology Letters, vol. 26, no. 5, pp. 469-472, 2014.

[18] S. Karman, S. Diah, and I. Gebeshuber, Bio-inspired polarized skylight-based navigation sensors: A review. Sensors, vol. 12, no. 11, pp. 14232-14261, 2012.

[19] J. Yang, T. Du, X. Liu, B. Niu, and L. Guo, Method and implementation of a bio-inspired attitude and heading reference system by integration of polarization compass and inertial sensors, IEEE Transactions on Industrial Electronics, 2019.

[20] B. Liu, Z. Fan, and X. Wang, Solar position acquisition method for polarized light navigation based on $\infty$ characteristic model of polarized skylight pattern, IEEE Access, 8, 56720-56729, 2020.

[21] C. Fan, X. Hu, J. Lian, L. Zhang, and X. He, Design and calibration of a novel camera-based bio-inspired polarization navigation sensor, IEEE Sensors Journal, vol. 16, no. 10, pp. 3640-3648, 2016.

[22] C. Fan, X. Hu, X. He, L. Zhang, and J. Lian, Integrated polarized skylight sensor and MIMU with a metric map for urban ground navigation. IEEE Sensors Journal, vol. 18, no. 4, pp. 1714-1722, 2017.

[23] C. Fan, X. Hu, X. He, L. Zhang, and Y. Wang, Multicamera polarized vision for the orientation with the skylight polarization patterns. Optical Engineering, vol. 57, no. 4, 043101, 2018.

[24] Y. Wang, X. Hu, J. Lian, L. Zhang, and X. He, Bionic orientation and visual enhancement with a novel polarization camera, IEEE Sensors Journal, vol. 17, no. 5, pp. 1316-1324, 2017.

[25] J. Dupeyroux, J. R. Serres, and S. Viollet, AntBot: a six-legged walking robot able to home like desert ants in outdoor environments, Science Robotics, vol. 4, eaau0307, 2019.

[26] J. Dupeyroux, S. Viollet, and J. R. Serres, An ant-inspired celestial compass applied to autonomous outdoor robot navigation, Robotics and Autonomous Systems, vol. 117, pp. 40-56, 2019.

[27] T. Labhart, The electrophysiology of photoreceptors in different eye regions of the desert ant, Cataglyphis bicolor. Journal of Comparative Physiology A, vol. 158, no. 1, pp. 1-7, 1986.

[28] J. Dupeyroux, S. Viollet, and J. R. Serres, Polarized skylight-based heading measurements: a bio-inspired approach, Journal of the Royal Society Interface, vol. 16, 20180878, 2019.

[29] T. M. Aycock, D. Chenault, A. Lompado, and J. L. Pezzaniti, Sky polarization and sun sensor system and method, U.S. Patent No. 9,423,484. Washington, DC: U.S. Patent and Trademark Office, 2016.

[30] J. Monnoyer, J. Dupeyroux, S. Viollet, and J. Serres. Dispositif de détection d'un cap d'un véhicule par détection de photons polarisés linéairement, French Patent No. FR3086088A1, March 2020. 